
\title{
$\mathrm{UNLV} \mid \underset{\text { LIBRARIES }}{\mathrm{UNIVRSTT}}$
}

10-1999

\section{Validity of the Independent-Particle Approximation in X-Ray Photoemission: The Exception, Not the Rule}

\author{
D. Hansen \\ University of Nevada, Las Vegas
}

Oliver Hemmers

University of Nevada, Las Vegas, Oliver.Hemmers@unlv.edu

H. Wang

University of Nevada, Las Vegas

Dennis W. Lindle

University of Nevada, Las Vegas, lindle@unlv.nevada.edu

P. Focke

University of Tennessee, Knoxville

Follow this and additional works at: https://digitalscholarship.unlv.edu/hrc_fac_articles

Part of the Atomic, Molecular and Optical Physics Commons, Inorganic Chemistry Commons, and the See next page for additional authors

\section{Repository Citation}

Hansen, D., Hemmers, O., Wang, H., Lindle, D. W., Focke, P., Sellin, I. A., Heske, C., Chakraborty, H. S., Deshmukh, P. C., Manson, S. T. (1999). Validity of the Independent-Particle Approximation in X-Ray Photoemission: The Exception, Not the Rule. Physical review A, 60(4), R2641-R2644.

https://digitalscholarship.unlv.edu/hrc_fac_articles/36

This Article is protected by copyright and/or related rights. It has been brought to you by Digital Scholarship@UNLV with permission from the rights-holder(s). You are free to use this Article in any way that is permitted by the copyright and related rights legislation that applies to your use. For other uses you need to obtain permission from the rights-holder(s) directly, unless additional rights are indicated by a Creative Commons license in the record and/ or on the work itself.

This Article has been accepted for inclusion in Environmental Studies Faculty Publications by an authorized administrator of Digital Scholarship@UNLV. For more information, please contact digitalscholarship@unlv.edu. 


\section{Authors}

D. Hansen, Oliver Hemmers, H. Wang, Dennis W. Lindle, P. Focke, I. A. Sellin, Clemens Heske, H. S. Chakraborty, P. C. Deshmukh, and S. T. Manson

This article is available at Digital Scholarship@UNLV: https://digitalscholarship.unlv.edu/hrc_fac_articles/36 


\title{
Validity of the independent-particle approximation in $\mathrm{x}$-ray photoemission: The exception, not the rule
}

\author{
D. L. Hansen, ${ }^{1}$ O. Hemmers, ${ }^{1}$ H. Wang, ${ }^{1}$ D. W. Lindle, ${ }^{1}$ P. Focke, ${ }^{2}$ I. A. Sellin, ${ }^{2}$ C. Heske, ${ }^{3}$ H. S. Chakraborty, ${ }^{4}$ \\ P. C. Deshmukh, ${ }^{4}$ and S. T. Manson ${ }^{5}$ \\ ${ }^{1}$ Department of Chemistry, University of Nevada, Las Vegas, Nevada 89154-4003 \\ ${ }^{2}$ Department of Physics and Astronomy, University of Tennessee, Knoxville, Tennessee 37996-1200 \\ ${ }^{3}$ Advanced Light Source, Lawrence Berkeley National Laboratory, Berkeley, California 94720 \\ ${ }^{4}$ Department of Physics, Indian Institute of Technology-Madras, Chennai 600036, India \\ ${ }^{5}$ Department of Physics and Astronomy, Georgia State University, Atlanta, Georgia 30303-3083
}

(Received 22 January 1999)

\begin{abstract}
A combined experimental and theoretical study of argon valence photoionization illustrates the discovery of the broad lack of validity of the independent-particle approximation (IPA) for x-ray photoemission. In addition to previously known breakdowns of the IPA, which are limited to high photon energies and regions very near threshold, the observed breakdown in photoionization at intermediate energies demonstrates generally that the IPA is valid only in very restricted domains. These restrictions are expected to be relevant throughout the periodic table, with consequences for a wide variety of applications. [S1050-2947(99)50810-X]
\end{abstract}

PACS number(s): 32.80.Fb, 32.80.Dz

One fundamental assumption commonly applied to manyelectron quantum systems is the one-electron or independentparticle approximation (IPA), in which effects of electronelectron interactions, i.e., electron correlation, are assumed to be negligible. Among the myriad of applications of the IPA is describing $\mathrm{X}$-ray interactions with matter in all its forms; the IPA is readily used as a basis for theoretical calculations and tabulations of a variety of x-ray-interaction parameters, such as total and partial cross sections, photon-scattering probabilities, and photoelectron angular distributions. With this central role in $\mathrm{X}$-ray science, it is important to determine the limits of the IPA or, equivalently, to identify the significance of electron-correlation effects to x-ray interactions. At present, it is a generally accepted axiom that the IPA is valid, particularly at energies well above threshold and away from inner-shell thresholds [1-7], except in certain well-defined regimes where electron correlation is known to be important. It is the purpose of this Rapid Communication to demonstrate that this notion is incorrect for $\mathrm{x}$-ray photoemission; the validity of the IPA is the exception, not the rule.

Atomic photoionization is an excellent "laboratory" which to study the effects of electron correlation, and thus the limits of the IPA, for two reasons. First, the coupling between the ionizing radiation and the target is weak [8], thus allowing unambiguous investigation of target dynamics. Second, because the target is simple and the forces are known, the process is amenable to detailed $a b$ initio theoretical calculation and the various approximations are quite assessable. Over the years a large number of theoretical and experimental studies have focused upon various aspects of correlation in the photoionization process.

Electron correlation in the form of interchannel coupling is simply configuration interaction among continuum wave functions corresponding to differing states of the residual ion, and has been found to be a crucial determinant of the photoionization process in a number of distinct situations. In the vicinity of outer-shell thresholds, weak channels can be dramatically altered by interchannel coupling [9]; the IPA sometimes fails to describe the situation even qualitatively. For example, the threshold behavior of the photoionization cross section of Xe $5 s$ is completely dominated by interchannel coupling with the $5 p$ and $4 d$ channels [10]. In small energy regions above inner-shell thresholds, the larger innershell cross section often affects outer-shell cross sections dramatically via interchannel coupling; many examples of this phenomenon are known [11]. And at high energies, where $n s$-subshell cross sections dominate, owing to the fact that the photoionization cross section for an $n l$ subshell falls off as $E^{(-7 / 2+l)}$, interchannel coupling significantly modifies the other $n l \quad(l \neq 0)$ subshell cross sections [12]. It is clear that the IPA breaks down in these well-defined energy regimes.

At intermediate energies, however, far above outer-shell thresholds and away from inner-shell thresholds, interchannel-coupling effects are thought to be small and the IPA is reckoned to be reasonably good. In this Rapid Communication, a combined theoretical and experimental study of valence photoionization of argon demonstrates that this is not the case; the IPA is invalid in a broad region of energy and subshell. This is of great interest, owing to the upsurge in activity in the field of atomic photoionization, spurred by the development of third-generation synchrotron-radiation sources [13], along with the importance of atomic photoionization in various applications, e.g., radiation physics and astrophysical modeling [14].

The experiments were performed at the Advanced Light Source (ALS) at Lawrence Berkeley National Laboratory (LBNL) on an undulator beamline 8.0 using a gas-phase time-of-flight (TOF) photoelectron-spectroscopy system designed specifically for soft-X-ray work at the ALS. A complete discussion of this apparatus is published elsewhere [15]. A key characteristic of the present measurements is that the TOF method can measure photoelectron peaks at many kinetic energies and at multiple emission angles simultaneously, permitting sensitive determinations of cross-section ratios and electron angular distributions with minimal experimental uncertainty. 
Calculations have been performed within the framework of the relativistic-random-phase approximation (RRPA) $[16,17]$. All relativistic single-excitation channels of $\mathrm{Ar}$ in the photon-energy range from threshold to $2 \mathrm{keV}$ were included. Note that, in addition to ground state-correlation, including the mixing of the initial $3 p^{6}$ state with the important $3 p^{4} 3 d^{2}$ configuration, as well as all two-electron promotions in the Ar-ion cores, the RRPA takes into account interchannel coupling among all of the single-excitation final-state channels. The calculation is performed in both length and velocity formulations, which must be equal in RRPA calculations; the equality of our length and velocity results to $\sim 1 \%$ demonstrates the numerical accuracy of the calculations, even at such high energies.

Our experimental and theoretical results for the $\operatorname{Ar} 3 s$ to $3 p$ cross-section ratio, $\sigma_{3 s} / \sigma_{3 p}$, are shown in Fig. 1 . The ratio is investigated, rather than the individual cross sections, because most experimental uncertainties cancel in the ratio, and it thus has fewer possible systematic errors than the relative cross sections. Four levels of calculation are also shown in Fig. 1, with the key distinction being whether coupling between the $3 s$ and $3 p$ channels is included (solid and dashed curves) or not included (dotted and dash-dotted curves). The dotted curve represents calculations including only ground-state correlation and coupling among channels arising from the same $n l$ subshell; these correlations are, however, unimportant far from threshold. Thus the dotted curve is almost indistinguishable from the IPA result (not shown), which we have also calculated. Of particular importance is the agreement of the various levels of theory with experiment. The two theoretical results that include $3 s-3 p$ interchannel coupling are in much better agreement with experiment than the pair of calculated results omitting this coupling. The former lie about $25 \%$ above experiment; the latter are off by almost a factor of 2 .

These results can be understood using basic perturbation theory. In the energy region of the measurements, the $\sigma_{3 s} / \sigma_{3 p}$ ratio is much less than unity; thus the $3 s$ dipole

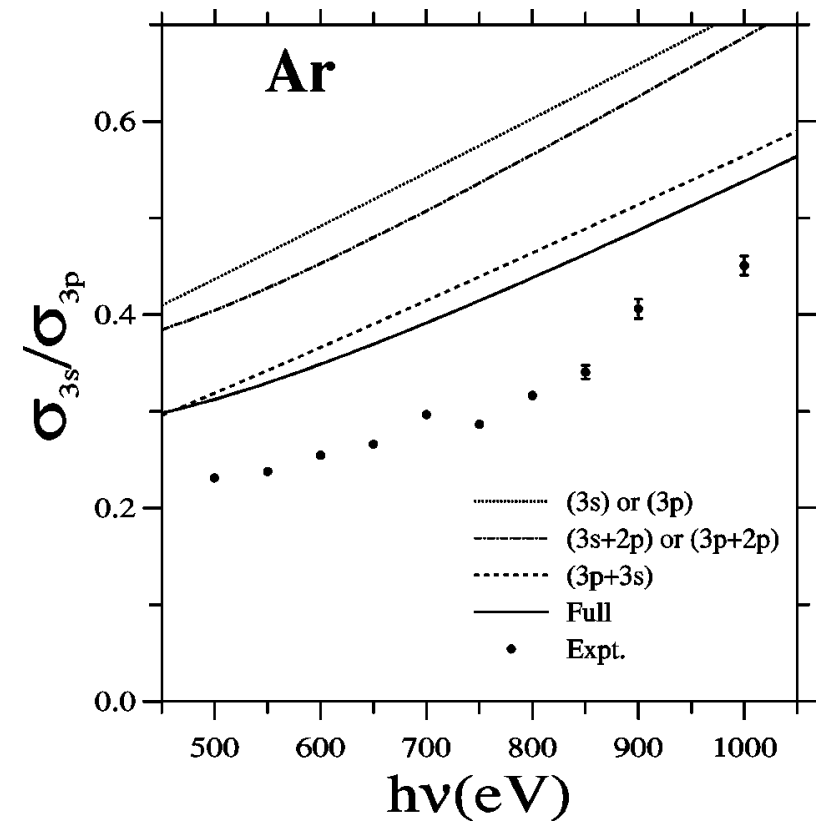

FIG. 1. Ratio of the $3 s$ to $3 p$ photoionization cross sections for Ar. The points are the present experiment. The theoretical results employed the RRPA formalism with the single excitation channels arising from $3 p, 3 s, 2 p, 2 s$, and $1 s$ coupled (solid curve); only $3 p$ and $3 s$ coupled (dashed curve); $3 p$ and $3 s$ only coupled to $2 p$ but not to each other (dot-dashed curve); and $3 p$ and $3 s$ uncoupled, essentially the IPA (dotted curve).

matrix element $M_{3 s \rightarrow k p}$ is significantly smaller than the dominant $3 p$ dipole matrix element $M_{3 p \rightarrow k d}$. Therefore, a small amount of mixing of the $3 p \rightarrow k d^{1} P$ final-state wave function with the $3 s \rightarrow k p^{1} P$, a continuum configuration interaction, can have a substantial influence on the latter. Using first-order perturbation theory, which should provide an excellent qualitative picture of the mixing, the perturbed wave function for the $3 s \rightarrow k p^{1} P$ final state $\Psi$, at energy $E$, is given by [18]

$$
\Psi_{3 s \rightarrow k p}(E)=\psi_{3 s \rightarrow k p}(E)+\int \frac{\left\langle\psi_{3 s \rightarrow k p}(E)\left|H-H_{0}\right| \psi_{3 p \rightarrow k^{\prime} d}\left(E^{\prime}\right)\right\rangle \psi_{3 p \rightarrow k^{\prime} d}\left(E^{\prime}\right) d E^{\prime}}{E-E^{\prime}},
$$

where $\psi$ represents the unperturbed wave functions, and the matrix element under the integral sign is the interchannel coupling matrix element of the perturbation, the total Hamilitonian $H$ minus the unperturbed Hamiltonian $H_{0}$. The $\psi$ 's are eigenfunctions of $H_{0}$. Note that an integral occurs in Eq. (1), rather than a sum, because we are dealing with continuum states. This integral has a singularity at $E=E^{\prime}$ and can be evaluated using the Cauchy principal value theorem [18]. If we define the unperturbed (IPA) dipole matrix element for channel $j$ as

$$
D_{j}(E)=\left\langle\psi_{i}|T| \psi_{j}(E)\right\rangle,
$$

with $\psi_{i}$ the initial-state wave function and $T$ the transition operator, the corrected dipole matrix element for the $3 s \rightarrow k p$ transition is given by

$$
M_{3 s \rightarrow k p}(E)=D_{3 s \rightarrow k p}(E)+\int \frac{\left\langle\psi_{3 s \rightarrow k p}(E)\left|H-H_{0}\right| \psi_{3 p \rightarrow k^{\prime} d}\left(E^{\prime}\right)\right\rangle D_{3 p \rightarrow k^{\prime} d}\left(E^{\prime}\right) d E^{\prime}}{E-E^{\prime}} .
$$


Because $M_{3 p \rightarrow k d}$ is the largest of the dipole matrix elements, it is not very much affected by the coupling, and is virtually the same as the unperturbed $D_{3 p \rightarrow k d}$. But, as long as the interchannel-coupling matrix element (the matrix element of $\left.H-H_{0}\right)$ is significant, the $3 s \rightarrow k p$ dipole matrix element $M_{3 s \rightarrow k p}$ is substantially modified from its unperturbed value.

It is well known that in the threshold region there is substantial interchannel coupling, and $M_{3 s \rightarrow k p}$ is significantly modified [9-11]. As discussed earlier, there are many cases reported of interchannel effects in the threshold region of outer and inner shells. The inner-shell cases are distinguished by broad resonancelike behavior in the outer-shell cross section just above the inner-shell threshold, but this interchannel-coupling effect dies out rather quickly with increasing energy. The interchannel-coupling matrix element is the key to this behavior. At an inner-shell threshold, the inner-shell photoelectron has zero energy and the continuum wave function is not very oscillatory, so the interchannelcoupling matrix element is significant. With increasing energy, the inner-shell continuum wave function becomes more oscillatory, but with a rather different period from the outershell continuum wave function, owing to the large difference in binding energy between inner and outer shells. This causes the interchannel-coupling matrix element to fall off rapidly with energy. This, in turn, diminishes the importance of interchannel coupling on the outer-shell cross section, as seen both experimentally and theoretically [11].

In contrast, interchannel coupling among channels with similar binding energies arising from subshells with similar spatial extent (subshells with the same principal quantum number, or for certain higher- $Z$ elements, some subshells with principal quantum numbers that differ by 1) is markedly different in one crucial aspect; because the channels have similar binding energies, they naturally have similar photoelectron energies. This means that the photoelectron continuum wave functions exhibit constructive interference over a broad range of energy so that the interchannel-coupling matrix element falls off very slowly with increasing energy. In addition, if the subshells from which the channels arise have similar spatial extent, they overlap significantly and the interchannel-coupling matrix element is large to begin with. These conditions lead to interchannel-coupling effects persisting very far from threshold, as demonstrated in Fig. 1.

To be sure it is indeed the smaller $3 s \rightarrow k p$ cross section that is altered by the interchannel coupling. We also have investigated the photoelectron angular-distribution asymmetry parameter $\beta$ for $3 p$ photoionization; we investigate $\beta$ because it can be measured with less absolute error than $\sigma$ and because it is generally more sensitive to interchannel coupling [19]. The results are shown in Fig. 2, where it is seen that all levels of calculation are quite close to each other and that our experimental results agree with all of them. Both agreement between experiment and theory, along with agreement among the various levels of theoretical results, reinforce the conclusion that it is primarily the $3 s$ transition that is altered as a result of the interchannel coupling, rather than the $3 p$.

Returning to $\sigma_{3 s} / \sigma_{3 p}$, although it has been demonstrated above that the calculations including interchannel coupling between the $3 s$ and $3 p$ transitions agree with experiment far better than calculations that exclude this coupling, there is

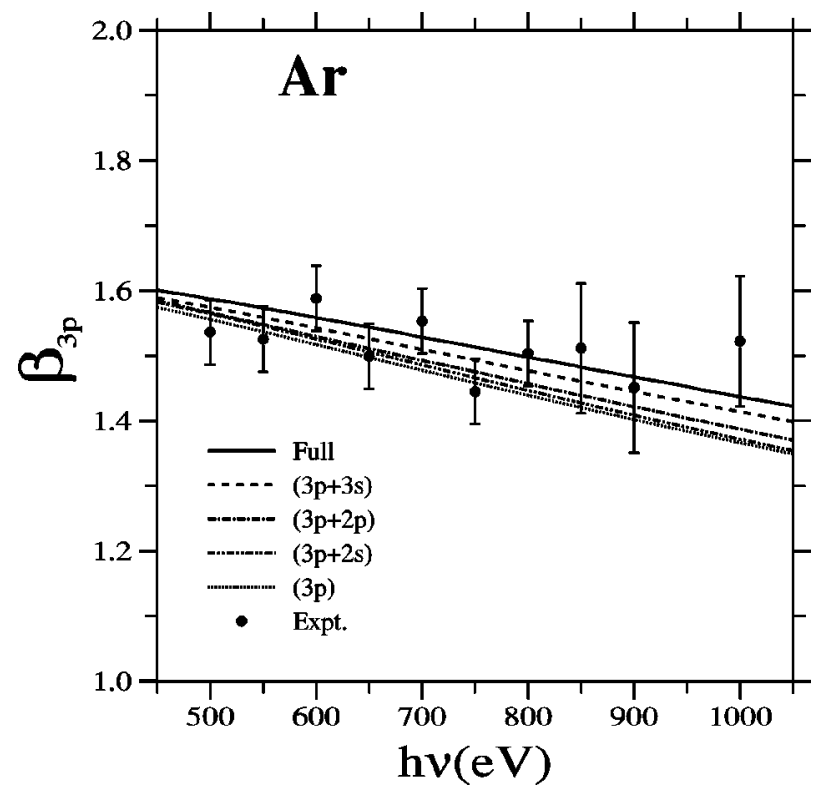

FIG. 2. Photoelectron angular-distribution asymmetry parameter $\beta$ for Ar $3 p$. The experimental points are compared to the four levels of theoretical results in Fig. 1, along with a calculation having $3 p$ coupled to $2 s$ and nothing else (dot-dot-dashed curve).

still a $\sim 25 \%$ difference between experiment and the full RRPA results in this range. It is very likely that the RRPA handles the important ground-state correlations correctly, as evidenced by good agreement with experiment in the energy region just above the lowest ionization potential in $\mathrm{Ar}$ and the other noble gases [20]. Interchannel coupling among the channels included also appears to be correct in a number of cases, both near threshold [9-11,20] and at high energy [12]. Thus the most plausible phenomenon to explain the discrepancy in this energy range seems to be interchannel coupling with channels omitted from the RRPA calculation.

In particular, two-electron excitations are not treated in the RRPA; correlation satellites arising from ionization of one $3 p$ electron and excitation of another, leaving $\mathrm{Ar}^{+}$in an excited state, are omitted. Interchannel coupling with satellite transitions is known to have a significant effect on Xe $5 s$ photoionization in the threshold region; the coupling of the $5 s$ cross section in the threshold region with the $5 p$ satellites is crucial for quantitative accuracy $[9,11]$. Thus it is reasonable that interchannel coupling of the $3 s$ channel with the many $3 p$ ionization plus excitation channels could account for the $25 \%$ discrepancy. This conclusion is further supported by the fact that in $\mathrm{Ne}$, where satellite transitions are known to be significantly less important than in Ar [10], excellent agreement is found between the cross-section ratio and RRPA calculations [12].

In conclusion, then, we have shown in a combined experimental and theoretical study that interchannel coupling between $3 s$ and $3 p$ photoionization channels in Ar dramatically alters the smaller $3 s$ cross section from the predictions of the IPA and other calculations that omit this coupling, by almost a factor of 2 in an energy region quite far from any thresholds. In addition, it is inferred that interchannel coupling of $3 s$ photoionization with $3 p$ satellite channels is important, although a more sophisticated calculation must be performed to confirm this conclusion. 
There is nothing special about Ar, and therein lies the importance of these results. In any case where there are two (or more) degenerate photoionization channels emanating from subshells with similar binding energies and spatial extent, the smaller cross section(s) will be significantly affected through interchannel coupling with the stronger channel(s). Furthermore, even the satellites of the stronger channel(s) can have noticeable effects on the channels with smaller cross section. Thus calculations that omit interchannel coupling are reliable only for the dominant channel in such a situation; weaker channels will not be predicted reliably by Hartree-Fock or any other IPA calculation for virtually all energies. We are thus led to the inescapable conclusion that the IPA is not valid for most subshells of most atoms at most energies. Finally, although the example presented was for an atom, these ideas should be equally valid for molecules, surfaces, and solids as well.

This work was supported by the National Science Foundation, NASA, and the U.S. Department of Energy (DOE). One of us (D.W.L.) is grateful for support from the UNLV Sabbatical Leave Program. The authors thank the staff of the ALS for their support. The ALS is supported by the Director, Office of Energy Research, Office of Basic Energy Sciences, Materials Science Division, of the U.S. DOE under Contract No. DE-AC03-SF00098 at LBNL.
[1] H. A. Bethe and E. E. Salpeter, Quantum Mechanics of Oneand Two-Electron Atoms (Springer-Verlag, Berlin, 1958), Sec. 71.

[2] J. W. Cooper, in Atomic Inner-Shell Processes, edited by B. Crasemann (Academic Press, New York, 1975), Vol. 1, p. 170.

[3] S. T. Manson and D. Dill, in Electron Spectroscopy: Theory, Techniques and Applications, edited by C. R. Brundle and A. D. Baker (Academic Press, New York, 1978), Vol. 2, pp. 186188.

[4] J. Berkowitz, Photabsorption, Photoionization and Photoelectron Spectroscopy (Academic Press, New York, 1979), p. 61.

[5] A. F. Starace, in Handbuch der Physik, edited by W. Mehlhorn (Springer-Verlag, Berlin, 1982), Vol. 31, p. 46.

[6] M. Ya. Amusia, Atomic Photoeffect (Plenum Press, New York, 1990), p. $70 \mathrm{ff}$.

[7] A. F. Starace, in Atomic, Molecular, \& Optical Physics Handbook, edited by G. W. F. Drake (AIP Press, Woodbury, NY, 1996), p. 305.

[8] H. A. Bethe and E. E. Salpeter, Quantum Mechanics of Oneand Two-Electron Atoms (Ref. [1]), p. 248ff.

[9] A. F. Starace, in Handbuch der Physik (Ref. [5]), pp. 1-121.

[10] V. Schmidt, Rep. Prog. Phys. 55, 1483 (1992).

[11] M. Ya. Amusia, Atomic Photoeffect (Ref. [6]), Chaps. 5 and 6.
[12] E. W. B. Dias, H. S. Chakraborty, P. C. Deshmukh, S. T. Manson, O. Hemmers, P. Glans, D. L. Hansen, H. Wang, S. B. Whitfield, D. W. Lindle, R. Wehlitz, J. C. Levin, I. A. Sellin, and R. C. C. Perera, Phys. Rev. Lett. 78, 4553 (1997).

[13] New Directions in Research with Third Generation, Soft X-Ray Sources, Vol. 254 of NATO Advanced Study Institute, Series E: Applied Sciences, edited by A. S. Schlachter and F. J. Wuilleumier (Kluwer, Dordrecht, The Netherlands, 1992).

[14] Applied Atomic Collision Physics, edited by H. S. W. Massey, E. W. McDaniel, and B. Bederson (Academic Press, New York, 1983), 5 volumes.

[15] O. Hemmers, S. B. Whitfield, P. Glans, H. Wang, D. W. Lindle, R. Wehlitz, and I. A. Sellin, Rev. Sci. Instrum. 69, 3809 (1998).

[16] W. R. Johnson and C. D. Lin, Phys. Rev. A 20, 964 (1979).

[17] W. R. Johnson C. D. Lin, K. T. Cheng, and C. M. Lee, Phys. Scr. 21, 409 (1980).

[18] U. Fano, Phys. Rev. 124, 1866 (1961).

[19] D. W. Lindle, L. J. Medhurst, T. A. Ferret, P. A. Heiman, M. N. Piancastelli, S. H. Liu, D. A. Shirley, T. A. Carlson, P. C. Deshmukh, G. Nasreen, and S. T. Manson, Phys. Rev. A 38, 2371 (1988).

[20] W. R. Johnson and K. T. Cheng, Phys. Rev. A 20, 978 (1979). 